VOL. 16 (1977), 49-53.

\title{
On common fixed point theorems
}

\section{S.P. Singh and B.A. Meade}

The aim of this paper is to extend a recent result of S.A. Husain and V.M. Sehgal [BuZZ. Austral. Math. Soc. 13 (1975), 261-267]. The condition that the function should be continuous in Husain and Sehgal, op. cit., is replaced by a semicontinuity condition. Moreover, a different proof is given, the last part of which requires no continuity or semicontinuity condition whatsoever.

In a recent paper Husain and Sehgal [1] proved some results on fixed and common fixed points under the condition of continuity of the function. In this paper the theorem has been proved under considerably weaker conditions.

Let $R^{+}$denote the set of nonnegative reals. Let $\psi$ denote a family of mappings such that each $\phi \in \psi, \phi:\left(R^{+}\right)^{5} \rightarrow R^{+}$, and $\phi$ is upper semicontinuous and nondecreasing in each coordinate variable. Also let $\gamma(t)=\phi\left(t, t, a_{1} t, a_{2} t, t\right)$ where $\gamma$ is a function $\gamma: R^{+} \rightarrow R^{+}$where $a_{1}+a_{2}=3$.

LEMMA 1. For every $t>0, \gamma(t)<t$ if and only if $\lim _{n \rightarrow \infty} \gamma^{n}(t)=0$.

Proof (Necessity). Since $\phi$ is upper semicontinuous, then $\gamma$ is upper semicontinuous. Assume $\lim _{n \rightarrow \infty} \gamma^{n}(t)=A$ where $A \neq 0$. Then

$$
A=\lim _{n \rightarrow \infty} \gamma^{n+1}(t) \leq \gamma \lim _{n \rightarrow \infty} \gamma^{n}(t)=\gamma(A)<A ;
$$

Received 4 August 1976. 
that is, $A<A$, a contradiction. Therefore $A=0$.

(Sufficiency). Since $\phi$ is nondecreasing, then $\gamma$ is nondecreasing. Given $\lim _{n \rightarrow \infty} \gamma^{n}(t)=0$, assume $\gamma(t)>t$ for some $t>0$. Then $\gamma^{n}(t)>t$ for some $t>0$ for $n=1,2,3, \ldots$. Thus $\lim _{n \rightarrow \infty} \gamma^{n}(t) \nrightarrow 0$, a contradiction. Also if $\gamma(t)=t$ for some $t>0$, then $\lim \gamma^{n}(t) \nrightarrow 0$. Hence, for all $t>0, \gamma(t)<t$. $n \rightarrow \infty$

THEOREM 1. Let $(X, d)$ be a complete metric space and let $S$ and $T$ be selfmoppings of $X$. Suppose there exists a $\phi \in \psi$ such that for alz $x, y \in X$,

(1) $d(S(x), T(y)) \leq \phi(d(x, y), d(x, S(x)), d(x, T(y)), d(y, S(x))$, $d(y, T(y)))$

where $\phi$ satisfies the condition: for any $t>0$,

$$
\phi\left(t, t, a_{1} t, a_{2} t, t\right)<t \text { where } a_{1}+a_{2}=3 .
$$

Then there exists a $z \in X$ such that $z$ is a unique common fixed point of $S$ and $T$.

Proof. Since $\gamma(t)=\phi(t, t, t, 2 t, t)<t$, then, by Lemma 1 , $\lim _{n \rightarrow \infty} \gamma^{n}(t)=0$. Now let $x_{0} \in X$ be any point. Then define a sequence of iterates $\left\{x_{n}\right\}$ in the following way: $x_{1}=S\left(x_{0}\right), x_{2}=T\left(x_{1}\right), x_{3}=S\left(x_{2}\right) \ldots x_{2 n}=T\left(x_{2 n-1}\right)$, $x_{2 n+1}=s\left(x_{2 n}\right), \cdots$.

Claim. $d\left(x_{1}, x_{2}\right) \leq d\left(x_{0}, x_{1}\right)$.

Assume $d\left(x_{0}, x_{1}\right)<d\left(x_{1}, x_{2}\right)$. Then using the triangular inequality, $d\left(x_{0}, x_{2}\right)<2 d\left(x_{1}, x_{2}\right)$. Let $r=d\left(x_{1}, x_{2}\right)$. Then $r \leq \phi\left(d\left(x_{0}, x_{1}\right), d\left(x_{1}, x_{2}\right), d\left(x_{1}, x_{1}\right), d\left(x_{0}, x_{2}\right), d\left(x_{0}, x_{1}\right)\right)<$ $<\phi(r, r, r, 2 r, r)$. 
By (2), $\phi(r, r, r, 2 r, r)<r$ and thus $r<r$, a contradiction:

therefore, $d\left(x_{1}, x_{2}\right) \leq d\left(x_{0}, x_{1}\right)$ and

$d\left(x_{1}, x_{2}\right) \leq \phi\left(d\left(x_{0}, x_{1}\right), d\left(x_{0}, x_{1}\right), 0\right.$,

$$
\left.2 d\left(x_{0}, x_{1}\right), d\left(x_{0}, x_{1}\right)\right) \leq \gamma d\left(x_{0}, x_{1}\right) .
$$

Similarly, $d\left(x_{2}, x_{3}\right) \leq \gamma\left(d\left(x_{1}, x_{2}\right)\right) \leq \gamma^{2}\left(d\left(x_{0}, x_{1}\right)\right)$ and in general,

$d\left(x_{n}, x_{n+1}\right) \leq \gamma^{n}\left(d\left(x_{0}, x_{1}\right)\right)$. Since $\lim _{n \rightarrow \infty} \gamma^{n}(t)=0$ for $t>0$;

therefore,

$$
\lim _{n \rightarrow \infty} d\left(x_{n}, x_{n+1}\right)=0 .
$$

In order to show $\left\{x_{n}\right\}$ is Cauchy, it is sufficient to show that $\left\{x_{2 n}\right\}$ is Cauchy. Suppose that $\left\{x_{2 n}\right\}$ is not a Cauchy sequence. Then there is an $\varepsilon>0$ such that for each integer $2 k, k \in I^{+}$, there exist integers $2 n(k)$ and $2 m(k)$ with $2 k \leq 2 n(k)<2 m(k)$ such that

$$
d\left(x_{2 n(k)}, x_{2 m(k)}\right)<\varepsilon .
$$

Let, for each integer $2 k, k \in I^{+}, 2 m(k)$ be the least integer exceeding $2 n(k)$ satisfying (4); that is, $d\left(x_{2 n(k)}, x_{2 m(k)-2}\right) \leq \varepsilon$ and

$$
d\left(x_{2 n(k)}, x_{2 m(k)}\right)>\varepsilon \text {. }
$$

Let $d_{n}=d\left(x_{n}, x_{n+1}\right)$. Then for each integer $2 k, k \in I^{+}$,

$$
\varepsilon<d\left(x_{2 n(k)}, x_{2 m(k)}\right) \leq d\left(x_{2 n(k)}, x_{2 m(k)-2}\right)+d_{2 m(k)-2}+d_{2 m(k)-1} \text {. }
$$

By (3) and (5),

$$
d\left(x_{2 n(k)}, x_{2 m(k)}\right) \rightarrow \varepsilon \text { as } k \rightarrow \infty .
$$

It follows from the triangular inequality that

$$
\left|d\left(x_{2 n}(k), x_{2 m(k)-1}\right)-d\left(x_{2 n(k)}, x_{2 m(k)}\right)\right| \leq d_{2 m(k)-1}
$$

and

$$
\left|d\left(x_{2 n(k)+1}, x_{2 m(k)-1}\right)-d\left(x_{2 n(k)}, x_{2 m(k)}\right)\right| \leq d_{2 m(k)-1}+d_{2 n(k)} .
$$


By (6), as $k \rightarrow \infty$,

$$
d\left(x_{2 n(k)}, x_{2 m(k)-1}\right) \rightarrow \varepsilon
$$

and

$$
d\left(x_{2 n(k)+1}, x_{2 m(k)-1}\right) \rightarrow \varepsilon
$$

Now let

$$
\begin{aligned}
& p(2 k)=d\left(x_{2 n(k)}, x_{2 m(k)}\right), \\
& q(2 k)=d\left(x_{2 n(k)}, x_{2 m(k)-1}\right),
\end{aligned}
$$

and

$$
r(2 k)=d\left(x_{2 n(k)+1}, x_{2 m(k)-1}\right) \text {. }
$$

Then

$$
p(2 k) \leq d_{2 n(k)}+d\left(s\left(x_{2 n(k)}\right), T\left(x_{2 m(k)-1}\right)\right) .
$$

By $(1)$,

$$
p(2 k)<a_{2 n(k)}+\phi\left(q(2 k), d_{2 n(k)}, p(2 k), r(2 k), d_{2 m(k)-1}\right) .
$$

Since $\phi$ is upper semicontinuous, as $n \rightarrow \infty$, it follows that $\varepsilon \leq \phi(\varepsilon, 0, \varepsilon, \varepsilon, 0) \leq \phi(\varepsilon, \varepsilon, \varepsilon, 2 \varepsilon, \varepsilon)<\varepsilon$, a contradiction. Therefore $\left\{x_{n}\right\}$ is a Cauchy sequence and hence by completeness, there is a $z \in X$ such that $x_{n} \rightarrow z$. We show that $z$ is a common fixed point of $S$ and $T$.

Since $\left\{x_{n}\right\}$ converges to $z$, therefore $\left\{x_{2 n}\right\}$ and $\left\{x_{2 n+1}\right\}$ both converge to $z$. Let $d(S(z), z)=\varepsilon>0$. Thus we have, for $n \geq N$, $d\left(x_{2 n}, z\right)<\frac{\varepsilon-\gamma(\varepsilon)}{4}$ and $d\left(x_{2 n}, x_{2 n+1}\right) \leq \frac{\varepsilon-\gamma(\varepsilon)}{4}$. Therefore using the triangular inequality,

$$
d\left(z, x_{2 n-1}\right) \leq d\left(z, x_{2 n}\right)+d\left(x_{2 n}, x_{2 n-1}\right)<\frac{\varepsilon-\gamma(\varepsilon)}{4}+\frac{\varepsilon-\gamma(\varepsilon)}{4}=\frac{\varepsilon-\gamma(\varepsilon)}{2} ;
$$

that is,

$$
d\left(z, x_{2 n-1}\right)<\varepsilon
$$

Using the triangular inequality and (7) we have 


$$
d\left(x_{2 n-1}, S(z)\right) \leq d\left(x_{2 n-1}, z\right)+d(z, S(z))<\varepsilon+\varepsilon ;
$$

that is, $d\left(x_{2 n-1}, S(z)\right)<2 \varepsilon$.

Now

$$
\varepsilon=d(S(z), z) \leq d\left(S(z), T\left(x_{2 n-1}\right)\right)+d_{2 n}+d\left(x_{2 n+1}, z\right) .
$$

By (1),

$\varepsilon \leq \phi\left(d\left(z, x_{2 n-1}\right), d(z, S(z)), d\left(z, x_{2 n}\right), d\left(x_{2 n-1}, S(z)\right), d_{2 n-1}\right)+$

$$
+d_{2 n}+d\left(x_{2 n+1}, z\right) \text {. }
$$

By $(7)$ and $(8), \varepsilon \leq \phi(\varepsilon, \varepsilon, \varepsilon, 2 \varepsilon, \varepsilon)+\frac{\varepsilon-\gamma(\varepsilon)}{4}+\frac{\varepsilon-\gamma(\varepsilon)}{4}$; that is, $\varepsilon \leq \gamma(\varepsilon)+\frac{\varepsilon-\gamma(\varepsilon)}{2}=\frac{\gamma(\varepsilon)+\varepsilon}{2}$. Since $\gamma(\varepsilon)<\varepsilon$, then $\frac{\gamma(\varepsilon)+\varepsilon}{2}<\varepsilon$; that is, $\varepsilon<\varepsilon$, a contradiction. Therefore $z=S(z)$. Similarly $z=T(z)$.

It remains to show that $z$ is a unique common fixed point. Let

$z \neq y$ be two common fixed points of $S$ and $T$. Then

$$
\begin{aligned}
d(z, y) & =d(S(z), T(y)) \leq \phi(d(z, y), d(z, S(z)), d(z, T(y)), d(y, S(z)), \\
& d(y, T(y))) \\
& =\phi(d(z, y), 0, d(z, y), d(x, y), 0)<\gamma(d(z, y))<d(z, y) .
\end{aligned}
$$

Therefore $z=y$ and $S$ and $T$ have a unique common fixed point.

Taking $\phi$ to be continuous we get a slightly revised version of the result of Husain and Sehgal [1] as a corollary.

\section{References}

[1] S.A. Husain and V.M. Sehgal, "On common fixed points for a family of mappings", BuzZ. Austral. Math. Soc. 13 (1975), 261-267.

Department of Mathematics, Statistics and Computer Science, Memorial University of Newfoundland,

St John's,

Newfound land,

Canada. 